Mon. Not. R. Astron. Soc. 000, 000-000 (0000) Printed 27 October $2018 \quad$ (MN LATEX style file v1.4)

\title{
Magnetic flares and the optical variability of the X-ray transient XTE J1118+480
}

\author{
A. Merloni ${ }^{1}$, T. Di Matteo ${ }^{2 \star}$ and A. C. Fabian ${ }^{1}$ \\ 1 Institute of Astronomy, Madingley Road, Cambridge, CBз OHA. \\ 2 Harvard-Smithsonian Center for Astrophysics, 60 Garden St., Cambridge, MA 02138, USA.
}

\begin{abstract}
The simultaneous presence of a strong quasi periodic oscillation of period $\sim 10$ seconds in the optical and X-ray lightcurves of the X-ray transient XTE J1118+480 suggests that a significant fraction of the optical flux originates from the inner part of the accretion flow, where most of the X-rays are produced. We present a model of magnetic flares in an accretion disc corona where thermal cyclo-synchrotron emission contributes significantly to the optical emission, while the X-rays are produced by inverse Compton scattering of the soft photons produced by dissipation in the underlying disc and by the synchrotron process itself. Given the observational constraints, we estimate the values for the coronal temperature, optical depth and magnetic field intensity, as well as the accretion rate for the source. Within our model we predict a correlation between optical and hard X-ray variability and an anticorrelation between optical and soft Xrays. We also expect optical variability on flaring timescales ( $\sim$ tens of milliseconds), with a power density spectrum similar to the one observed in the X-ray band. Finally we use both the available optical/EUV/X-ray spectral energy distribution and the low frequency time variability to discuss limits on the inner radius of the optically thick disc.
\end{abstract}

Key words: accretion, accretion discs - magnetic fields - radiation mechanisms: thermal - binaries: general - stars: individual: XTE J118+480

\section{INTRODUCTION}

The newly discovered transient X-ray source XTE J1118+480 (Remillard et al. 2000), has already shown a number of remarkable properties. It was discovered as a weak source with the RXTE All-Sky Monitor on March 29, 2000 at high galactic latitude $\left(b \sim 62^{\circ}\right)$. Subsequent RXTE pointed observations revealed an energy spectrum typical of black hole candidates in their hard state, showing a prominent power-law component in its X-ray spectrum with a photon index of about 1.8 up to at least $30 \mathrm{keV}$. The source was also observed on March 26 in hard X-rays by BATSE, up to $120 \mathrm{keV}$ (Wilson \& McCollough 2000). The 13th magnitude optical counterpart, discovered by Uemura, Kato \& Yamaoka (2000), exhibit a spectrum fairly typical of X-ray Novae in outburst (e.g. Garcia et al. 2000).

Being at such an high galactic latitude, the source suffers from low interstellar absorption, and consequently has also been observed in the extreme ultra violet (EUV) by the Extreme Ultraviolet Explorer (EUVE). The EUVE spectrum and fluxes derived by Hynes et al. (2000) however are ex-

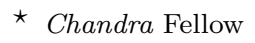

tremely sensitive to the details of the absorbing column and are therefore subject to large uncertainties.

A strong QPO feature with a frequency $\nu \sim 0.08 \mathrm{~Hz}$ has been found in the X-ray power density spectrum (PDS) of the source in the first month of the outburst (March 29 - May 4, see Revnitsev, Sunyaev \& Borozdin 2000). More surprisingly, the optical lightcurves also show strong flickering on timescales of a few seconds or faster and a prominent quasi periodic feature with a frequency in agreement with that of the X-ray QPO. Subsequent HST, RXTE and ASCA observations in the optical/UV and X-ray band respectively, have also shown that the QPO is drifting to higher frequencies systematically in both bands, from $\nu \sim 0.08 \mathrm{~Hz}$ to $\nu \sim 0.11 \mathrm{~Hz}$ later in the month (Haswell et al. 2000b; Yamaoka, Ueda \& Dotani 2000; Patterson private communication).

The strikingly similar variability properties observed in both bands suggest that at least a fraction of the optical/UV flux from the object should be produced in the same region of the accretion flow where the X-rays are produced and hence reflect a different aspect of the same phenomenon. This implies that the modulated optical/UV flux cannot be produced by thermal emission from the accretion disc itself; the optical/UV flux from a stellar mass black hole arises

(C) $0000 \mathrm{RAS}$ 
from the outer region of the disc (radii greater than hundreds of Schwarzchild radii) where the X-ray emission is negligible.

Here we show that the presence of such optical/UV variability in the observations of XTE J1118+480 argues for the presence of significant thermal synchrotron radiation from a magnetically dominated corona (or possibly from an advection dominated accretion flow, ADAF, occupying the inner parts of the flow).

The only other object ever observed to show similar short timescale variability, in both the optical and X-ray bands, is the black hole candidate GX 339-4 in its hard state (Motch et al 1982). For the case of GX 339-4, typically brighter than XTE J1118+480 in X-rays, it was also suggested that thermal synchrotron emission should provide a significant contribution to the optical band (Fabian et al. 1982; Di Matteo, Celotti \& Fabian 1999).

\section{CYCLO-SYNCROTRON EMISSION FROM A MAGNETIC CORONA}

\subsection{The Magnetic field in the corona}

It is now well established that magnetic stresses in accretion discs are likely to be responsible for the transfer of angular momentum (see Balbus \& Hawley 1998 for a review). Magneto-rotational instabilities can drive turbulence by amplifying the seed magnetic fields on roughly Keplerian timescales; the rate at which magnetic energy is built up is fast enough to explain the bulk of energy release in an accretion disk as magnetic dissipation. This supports the idea that accretion disc coronae, the loci where the hard X-ray emission is produced, are highly magnetic and form by buoyancy of the strong magnetic fields amplified in the disk (e.g. Galeev, Rosner \& Vaiana 1979). This picture is supported by numerical simulations (Miller \& Stone 2000) which show that, when weak $B$ fields are amplified via MHD turbulence in the disk, only a fraction $\sim H / R<1$ of the energy is dissipated locally while the rest escapes and forms a strongly magnetized corona above the disc.

In any such models, the magnetic field in the corona is likely to be strongly inhomogeneous and to dissipate energy in localized active regions. Following Di Matteo, Celotti \& Fabian $(1997 ; 1999)$, we assume that a significant fraction, $f$, of the accretion power, $L=\dot{m} L_{\mathrm{Edd}}$, is accumulated in the corona while the remaining fraction $(1-f)$ is dissipated internally in the disc. The magnetic field strength, $B$, in the magnetic flux tubes rising from the disk can be higher than the values implied by equipartition with radiation energy density. In fact, if the active region is powered by the release of magnetic energy, its dissipation velocity is $\sim v_{\mathrm{A}}$, the Alfven speed (see e.g. Di Matteo 1998). The energy stored in the field is therefore a fraction $v_{A} / c \sim 0.1$ of that of the radiation (Di Matteo, Blackman \& Fabian 1997). Based on similar arguments Haardt, Maraschi \& Ghisellini (1994) showed that the magnetic energy is released on typical timesclales $t_{0} \sim 10 R_{\mathrm{b}} / c$, where $R_{\mathrm{b}}$ is the size of the active region. This leads to a magnetic field strength,

$\frac{B^{2}}{8 \pi} \frac{v_{\mathrm{A}}}{c} \approx \frac{9 f L}{16 \pi N\left(r_{\mathrm{b}} R_{\mathrm{s}}\right)^{2} c}$

where $N$ is the number of active regions and $r_{\mathrm{b}}$ their dimen- sion in units of $R_{\mathrm{s}}=2 G M / c^{2}$, the Schwarzschild radius for a given central mass $M=m \mathrm{M}_{\odot}$. This implies a value

$B \approx 10^{8} \frac{1}{r_{\mathrm{b}}}\left(\frac{\dot{m} f c}{N m v_{\mathrm{A}}}\right)^{1 / 2} \mathrm{G}$

for the estimated magnetic field in the inner part of the accretion flow.

\subsection{Synchrotron emission}

In the hot $(T \sim 50-200 \mathrm{keV})$, highly magnetized, coronal plasma the majority of the electrons are thought to be thermal and are expected to produce synchrotron emission, and Compton scatter the softer photons produced both by dissipation in the underlying accretion disk and by the synchrotron processes themselves.

In a thermal plasma, optically-thin synchrotron emission rises steeply with decreasing frequency. Under most circumstances the emission becomes self-absorbed and gives rise to a black-body spectrum below a critical frequency: $\nu_{\mathrm{c}} \approx 2.7 \times 10^{14} \tau^{0.05}\left(\frac{T}{10^{9} \mathrm{~K}}\right)^{0.95}\left(\frac{B}{10^{6} \mathrm{G}}\right)^{0.91} \mathrm{~Hz}$ (see Wardziński \& Zdziarski 2000), where $T$ is the coronal temperature and $\tau$ the scattering optical depth. Above this frequency, which is right in the optical/UV band for typical parameter of $\mathrm{X}$ ray transient sources and magnetic fields $B \sim 10^{6}-10^{7} \mathrm{G}$, the cyclo-synchrotron emission decays exponentially as expected from a thermal plasma, due to the superposition of cyclotron harmonics.

In an active region above an accretion disc, the relative importance of the cyclo-synchrotron emission (and its Comptonization) as opposed to the Comptonization of the external photon field has to be estimated by comparing the relevant timescales for the processes. Such comparisons have shown that, as the synchrotron emission is heavily self-absorbed, the internal energy density of the synchrotron radiation $U_{\text {rad, int }}<B^{2} / 8 \pi \lesssim U_{\text {rad, ext }}$, where $U_{\text {rad,ext }}=(1-f) L / 4 \pi c R_{\text {disc }}^{2}$ is the 'external' energy density of the radiation emerging from the disc. Consequently (and in accordance to standard models) most of the X-ray emission in the soft and hard states of GBH is usually due to Comptonization of the external photon fields (and not of the synchrotron component; Di Matteo, Celotti \& Fabian 1997; 1999; Wardziński \& Zdziarski 2000). Nevertheless, in the hard states of GBH the disk blackbody component, which dominates the X-ray emission in the soft state, often disappears or cools down significantly, and the synchrotron component is likely to contribute at least in the optical/UV band.

Here we show that thermal synchrotron emission can be an important radiation process in the case of XTE $\mathrm{J} 1118+480$.

\section{APPLICATION TO XTE J1118+480}

\subsection{Spectral modeling}

Hubble Space Telescope observations of XTE J1118+480 (Haswell et al. 2000a) were made on 2000 April 8, at the end of the rising phase of the outburst. The data indicate a flux density at $1500 \AA$ of $3 \times 10^{-13} \mathrm{ergs} \mathrm{cm}^{-2} \mathrm{~s}^{-1} \AA^{-1}$ and a spectrum between 1200 and $8000 \AA$ slightly flatter than 
$F_{\nu} \propto \nu^{1 / 3}$. On the other hand, unfiltered CCD fast photometry (Haswell et al. 2000b; Patterson 2000 has revealed optical flickering of $\pm 0.2 \mathrm{mag}$ on timescales of seconds, which correspond to $\sim 20$ per cent of the optical flux. As the optical variability is strongly modulated by a QPO with a frequency comparable (if not the same) to that of the X-ray one, it has to be produced where most of the X-rays are produced, namely in the inner part of the accretion flow.

We assume that this rapid variability is due to self absorbed cyclo-synchrotron (CS) emission. To explicitly show the various parameter dependences of the synchrotron emission we write the flux at the peak frequency $\nu_{\mathrm{c}}$ as (Di Matteo, Ce otti \& Fabian 1997

$$
\begin{aligned}
F_{\mathrm{CS}} & =2 \pi m_{e} \nu_{c}^{3} \theta r_{\mathrm{b}}^{2} N\left(\frac{R_{\mathrm{S}}}{D}\right)^{2}\left[\frac{1}{1+t_{\mathrm{CS}} / t_{\mathrm{iC}}}\right] \\
& \simeq 5.7 \times 10^{-14} \theta r_{\mathrm{b}}^{2} N\left(\frac{\nu_{c}}{10^{15}}\right)\left(\frac{m}{d}\right)^{2} \\
& \times\left[\frac{1}{1+3.2 \times 10^{2} \tau U_{\mathrm{rad}}\left(\frac{10^{15}}{\nu_{c}}\right)^{3}}\right]
\end{aligned}
$$

where $d$ is the distance of the source in kiloparsecs, $\theta=$ $k T / m_{e} c^{2}$ the dimensionless temperature, $U_{\text {rad }}=U_{\text {rad,int }}+$ $U_{\text {rad,ext }}$ and the ratio of the relevant timescales for the cyclo-synchrotron and inverse Compton emission determines which process dominates in an active (see Section 2.2). Under the assumption that at least one fourth of the flux at $\nu=\nu_{c}=10^{15} \mathrm{~Hz}$ is due to CS, we deduce that the intrinsic dissipation in the accretion disc has to be fairly small $\left(\dot{m}(1-f) \sim 10^{-3}\right)$ implying a magnetic field intensity $B \simeq 2 \times 10^{6} \mathrm{G}$.

We model the spectrum of the source following the work of Di Matteo, Celotti \& Fabian (1999). The main feature of the model is that it considers reprocessing of coronal radiation in the accretion disc not only according to the active blob size but also according to their height above the disc. The temperature and the optical depth in the corona are constrained from the observed slope of the power-law in the X-ray spectrum $\left(\alpha \simeq 0.8 \propto(\tau \theta)^{-1}\right.$; e.g. Wardziński \& Zdziarski 2000). The spectrum is calculated self-consistently for every annulus of radius $R=r R_{\mathrm{S}}$ and width $d R \ll R$ and integrated over radius from $r=3$ to $r=1000$. We take into account all the relevant radiative processes and rescale them opportunely according to the ratios of their typical cooling timescales which are obtained by integrating each spectral component (more accurately than in Eq. 3)

In all cases we postulate that the accretion disc extends down to the innermost stable orbit of a non-rotating black hole $\left(R_{\text {in }}=3 R_{\mathrm{S}}\right)$, so that both the optical and X-rays are produced where most of the energy is dissipated. The number of active regions at any given annulus is calculated as in Haardt, Maraschi \& Ghisellini (1994), following their discussion on the timescales over which the magnetic field is amplified in the disc $\left(t_{\mathrm{amp}} \sim t_{\mathrm{Kep}}\right)$ and released in the corona $\left(t_{\text {rel }} \sim t_{0}\right.$, see section 2.1). We obtain $d N=9.5 r^{-3 / 2} d r$, so that the total number of flares active at any time $N \simeq 10$, as usually assumed from variability arguments. The mass is fixed to 10 solar masses.

In Figure 1 we show an illustration of two model spectra for the recent optical and X-ray observations of XTE $\mathrm{J} 1118+480$. They are not to be regarded as detailed fits to the data, rather as indicative of the physical properties of the source implied by the model. In particular, we do not attempt to fit the optical continuum with a realistic model for the outermost parts of the accretion disc, our main interest being to put constraints on the nature of the inner accretion flow. The relatively high optical-to-X-ray flux ratio for the source imposes the major constraints on the model. In order for both the synchrotron component to be important in the optical band and the Comptonized flux not to exceed the observed limit in the hard X-ray band $U_{\text {rad, ext }}$, the external radiation energy density intercepting the active region (see Eq. 3) has to be lower than $(1-f) L /\left(4 \pi R_{\text {disc }}^{2} c\right)$.

The first spectrum (case a, solid lines) is obtained assuming static dissipation regions at fixed height. For $r_{\mathrm{b}}=3$ we have to allow the reconnection sites to be above the accretion disc at a height of $\sim 4-5 r_{\mathrm{b}}$. The need for such aspect ratio of the active region is just an indication that the soft radiation field seen by the flaring region has been reduced.

As expected from the simple scaling arguments of Eqs. (2) and (3), we obtain a relatively low accretion rate $(\dot{m} \simeq 0.01)$ and a high value of the fraction of the power dissipated in the corona $(f \simeq 0.97)$, both consistent with the source being in its hard state. However, in this geometry $f$ is not a relevant parameter because the hard X-ray radiation illuminates the disk and a substantial fraction of it $(\lesssim 0.5$, depending on the disc albedo) is reprocessed and thermalized giving rise to a hotter blackbody-like component roughly similar to that obtained for lower values of $f$.

In Figure 1 (case b, dashed lines) we illustrate an alternative way to reduce the contribution from this thermal component in the soft X-ray band and enhance the the cyclosynchrotron emission in the optical/UV band. Following Beloborodov (1999), we allow for bulk relativistic motion of the emitting coronal plasma away from the disc. Due to Doppler boosting the radiation from a reconnection site towards the disc is reduced. This is equivalent, for our purpose, to the assumption that the coronal emission is anisotropic: for $v / c \sim 0.3$, roughly 90 per cent of it is emitted upwards and only the remaining $\sim 10$ per cent impinges on the underlying disc.

Finally, the values of temperature and optical depth of the active regions we derive are, respectively, $\theta \simeq 0.3$ and $\tau \simeq 0.6$ and are almost equal in the two cases. The distance we obtain is of the order of $D=0.4 \mathrm{kpc}$, as expected for a high latitude galactic source.

\subsection{The inner disc}

Hynes ey al. (2000) have recently reported on EUV observations of XTE J1118+480 and have shown that the relatively low EUV flux may be inconsistent with an optically thick disc extending down to radii $r_{\text {in }} \lesssim 1000$. However EUV observations are extremely sensitive to the assumed absorption and the derived limits on $r_{\text {in }}$ highly uncertain. This is further emphasized by recent $A S C A$ observations which appear to show a slight soft excess below $2 \mathrm{keV}$ (Yamaoka et al. 2000) well fitted by a multicolour disc of temperature $0.2 \pm 0.1$ $\mathrm{keV}$. Because of the inconsistency between the ASCA and EUVE measurements we did not use the EUVE data as a further constraint for our model.

However, in accordance with the requirements discussed 


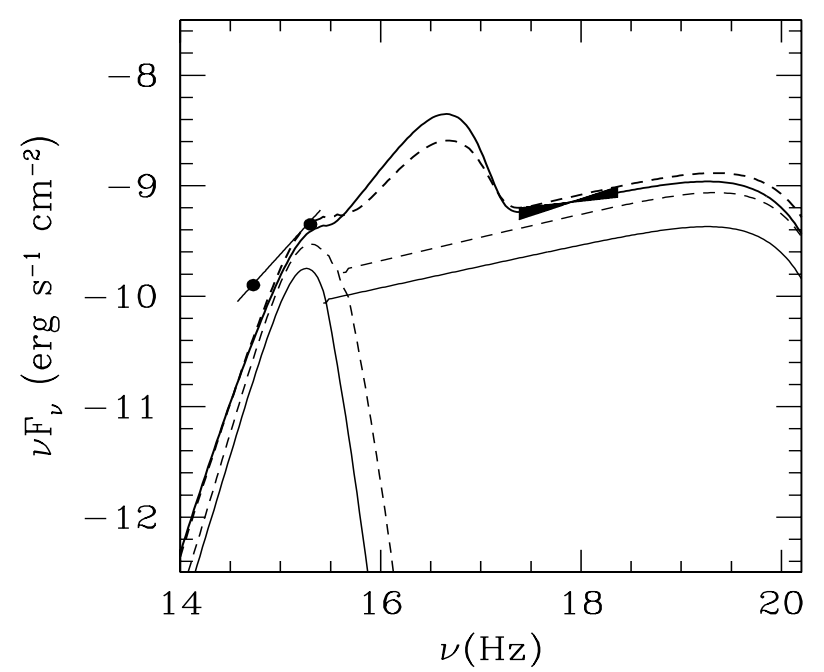

Figure 1. Representative model predictions for the spectral energy distribution of XTE J1118+480. The points represent optical (V band) (Uemura et al. 2000), near UV (Haswell et al. 2000a) and X-ray (Remillard et al. 2000, Yamaoka et al. 2000) observations of the source during its outburst. The two thicker lines represent models with (case a, solid line) and without (case $\mathrm{b}$, dotted lines) bulk relativistic motion of the active regions. The parameters are very similar for the two cases: for the active region parameters we obtain, respectively $\tau=0.61, \theta=0.3$, $r_{\mathrm{b}}=2.9, h=5.3 r_{\mathrm{b}}$ (case a) and $\tau=0.56, \theta=0.33, r_{\mathrm{b}}=3.2$, $h=4 r_{\mathrm{b}}$ (case b). We have assumed a mass of $10 \mathrm{M}_{\odot}$, and we estimate a distance of $0.4 \mathrm{kpc}$; the accretion flow parameters are: $\dot{m}=0.01$ and $f=0.97$. The self absorbed cyclo-synchrotron emission and its Comptonization are plotted (thinner lines). The optically thick part significantly contributes to the optical emission, together with the expected (non-varying) emission from the outermost parts of the accretion disc, while the X-ray are mainly produced by Comptonization of disc photons.

above, most objects observed in their hard/low state often show either no or very little evidence for soft blackbody emission or strong reflection features (i.e., the backscattered emission from the putative accretion disk). Based on this, it has therefore been argued that, in the hard state, geometrically thin discs may not extend down to the innermost stable orbit but are truncated at tens or hundreds of Schwarzchild radii. (Gierlińsky et al. 1997; Poutanen \& Coppi 1998; Zdziarski et al. 1998; Done \& Życki 1998; Esin et al. 1997; Wilms et al. 1999 and references therein).

If the disc does not extends down to the innermost stable orbit, then $U_{\text {rad,ext }}$ is suppressed and the relative importance of the synchrotron component (see Eq. 3) increases, as required by the data. Therefore, a central advection dominated (ADAF) component of the flow extending out to hundreds or thousands of Schwarzschild radii and surrounded by an optically thick accretion disc may also fit the spectrum reasonably well (see e.g. Esin et al. 1998). The information provided by temporal studies are then crucial for constraining the geometry of the inner disk, in particular optical$\mathrm{X}$-rays cross-correlation and time lag analysis for the QPO component.

\subsubsection{The $0.1 \mathrm{~Hz} Q P O$}

Psaltis, Belloni \& van der Klis (1999) have suggested that the frequencies of the periodic features in the PDS of black hole candidates (which can appear more or less broadened) follow a quite tight correlation that encompasses also neutron star sources, and therefore has to be related to the properties of the accretion flow. Recently Psaltis \& Norman (2000) have proposed a model in which the QPOs are produced in a narrow ring in the accretion disc where a discontinuity in some properties of the flow occurs. The frequencies themselves are essentially determined by the relativistic proper frequencies of the system (Stella, Vietri \& Morsink 1999; Merloni et al. 1999). Assuming such a model, the data can be used to constrain the position of the flow discontinuity (Di Matteo \& Psaltis 1999).

The published PDS of XTE J1118+480 strongly resembles that of GX 339-4 in the hard state (see Nowak 2000 for a discussion of the various variability components). Apart from the strongly peaked QPO at $0.08 \mathrm{~Hz}$, it exhibits a broader feature around $1 \mathrm{~Hz}$. Plotted one against the other, these frequencies fall remarkably well on the correlation presented by Psaltis, Belloni \& van der Klis (1999). This would suggest that the low frequency QPO is analogous to the $\mathrm{HBO}$ in neutron stars and is related to twice the nodal precession general-relativistic frequency of a perturbed orbit around the compact object. The value of the transition radius, where the modulation is produced, depends on the spin of the black hole, but is limited in the range $25 \gtrsim R_{t} / R_{\mathrm{S}} \gtrsim 6$ for a $10 \mathrm{M}_{\odot}$ black hole with dimensionless angular momentum $0.99 \gtrsim a \gtrsim 0.01$. This provides an upper bound for the inner extent of the geometrically thin accretion disc, which is in conflict with the limits derived from EUVE observations (Hynes et al. 2000) and significantly reduces the relevance of a central ADAF component, favoring instead models discussed in Section 3.1. We note, however, that if the observed QPO is associated with the HBO frequency it is not clear why the expected modulation at the keplerian frequency at the same radius (with frequency $\nu_{\text {Kep }} \sim 30 \mathrm{~Hz}$ ) is not directly observed, even though it should have a higher quality factor (although, see e.g. Nowak 2000).

Alternatively, we can ignore the very broad feature in the PDS at $\sim 1 \mathrm{~Hz}$ and consider the sharp low frequency QPO as due to Keplerian modulation of the flow far away from the source. This would imply a transition radius $R_{t} \simeq$ $500 R_{\mathrm{S}}$. Although this interpretation for the observed variability is less plausible, it may be in better agreement with the implied EUV fluxes and possibly with the presence of a central ADAF component. However, in this case it remains to be explained how the modulation propagates inward in the advective flow without loss of coherence, given that, in the ADAF model, the bulk of the optical and X-ray are produced in the inner part of the flow, where the magnetic field is high enough $\left(B_{A D A F} \sim 10^{7} \alpha_{0.1}^{-1 / 2} m_{10}^{-1 / 2} \dot{m}_{0.01}^{1 / 2} r_{3}^{-5 / 4} \mathrm{G}\right)$ to power the cyclo-synchrotron emission, which is then Componized to give rise to the high energy spectrum (see e. g. Narayan \& Yi 1995; Quataert \& Narayan 1999).

\section{DISCUSSION}

We have applied a model for the emission from a highly magnetic, structured corona to the optical and X-ray ob- 
servations of the newly discovered X-ray transient XTE $\mathrm{J} 1118+480$.

This source is unique for a number of reasons. It is located at high galactic latitude and has a very high opticalto-X-ray flux ratio. The source is observed in the typical black hole candidates hard state (photon index $\Gamma \simeq 1.8$ ). Given the optical/UV to X-ray flux ratio we have derived constraints for the approximate size, optical depth and magnetic field strengths of the coronal active regions.

The main point of our work is that the simultaneous presence of a strong quasi periodic feature in the optical and X-ray lightcurves clearly suggests that the fluxes in the two bands both originate from the same region in the inner part of the accretion flow. Self-absorbed cyclo-synchrotron emission is the natural candidate to explain the optical variability. Such emission is expected in any magnetic corona model, and the inferred magnetic field value $\left(B \approx 2 \times 10^{6}\right.$ $\mathrm{G})$ is the one predicted to arise when the source is in the hard state (low value of $\dot{m}$ and high value of $f$ ).

Our model is not unique. The relatively high opticalto-X-ray flux ratio observed can be explained either by a strong, structured magnetic corona with a relatvely high scaleheight, or by a central ADAF surrounded by an optically thick accretion disc. We have shown that even if cyclo-synchrotron emission plays an important role in the optical band in both cases, in our model the X-ray photons are mainly produced via inverse Compton scattering of the soft disc photons, while in the ADAF model the CS emission itself acts as source field. We gave an example of how variability data can be used to further discriminate between the models.

Independently of the dominant source of soft photons for Comptonization, we expect that any (small) variation in the physical parameters characterizing the active coronal regions (either in temperature or optical depths) driven by a flow discontinuity in the disk should modulate the emission simultaneously in the optical (via the CS emission) and in the X-ray band (via inverse Compton emission). In particular, any increase (at a level of 20 per cent or so, as required) in temperature or density in the corona will cause an increase in the cyclo-synchrotron component at optical frequencies, while at the same time it will make the X-ray spectrum harder. From any such model we then expect a correlation between the optical and hard K-ray variabitity but an andation has been observed for the case of GX 339-4; see Motch et al. 1982). Our model also predicts optical variability on flaring timescales ( $\sim$ tens of milliseconds), with a PDS similar to the one observed in X-rays. Furthermore the variable optical component is expected to drop out quickly at low frequencies $\nu<\nu_{\mathrm{c}}$ due to the fast decline of the synchrotron emission in the Rayleigh-Jeans regime. Time-resolved spectroscopy observations of such behaviour would support this model and allow us to place strong constraints on the magnetic field strength in the corona.

\section{ACKNOWLEDGMENTS}

We thank Annalisa Celotti for many useful suggestions and comments on the manuscript and Joe Patterson for useful informations on the optical variability. This work was done in the research network 'Accretion onto black holes, compact stars and protostars', funded by the European Commission under contract number ERBFMRX-CT98-0195. AM and ACF thank the PPARC and The Royal Society for support, respectively. TDM acknowledges support for this work provided by NASA through Chandra Fellowship grant number PF8-10005 awarded by the Chandra Science Center, which is operated by the Smithsonian Astrophysical Observatory for NASA under contract NAS8-39073.

\section{REFERENCES}

Balbus, S. A. \& Hawley, J. F., 1998, Rev. Mod. Phys. 70, 1.

Di Matteo, T., 1998, MNRAS, 299, L15.

Di Matteo, T., Blackman, E. G. \& Fabian, A. C., 1997, MNRAS, 291, L23.

Di Matteo, T., Celotti, A. \& Fabian, A. C., 1997, MNRAS, 291, 805.

Di Matteo, T., Celotti, A. \& Fabian, A. C., 1999, MNRAS, 304, 809.

Di Matteo, T. \& Psaltis, D., 1999, ApJ, 526, L101.

Done, C., \& Zycki, P. T., 1999, MNRAS, 305, 457.

Fabian, A., Guilbert, P. W., Motch, C., Ricketts, M., Ilovaisky, S. A., Chevalier, C., 1982, A\&A, 111, L9.

Galeev, A. A., Rosner, R. \& Vaiana, G. S., 1979, ApJ, 229, 318.

Garcia M., Brown W., Pahre M., J. McClintock 2000, IAUC 7392.

Gierliński, M., Zdziarski, A. A., Done, C., Johnson, W. N., Ebisawa, K., Ueda, Y., Phlips, F., 1997, MNRAS, 288, 958.

Haardt, F., Maraschi, L. \& Ghisellini, G., 1994, ApJ, 432, L95.

Haswell, Hynes, R. I. \& King, A. R., 2000a, IAUC 742

Haswell, C. A., Skillman, D., Patterson,J., Hynes, R. I., Cui, W., 2000b, IAUC 7427

Hynes, R. I., Mauche, C. W., Haswell, C. A., Schrader, C. R. . Cui, W., Chaty, S., 2000, submitted to ApJL, astro-ph/0005398.

Merloni, A., Stella, L., Vietri, M., Bini, D., 1999, MNRAS, 304, 155.

Miller, K. \& A.Stone, J. M., 2000, ApJ, 534, 398.

Motch, C., Ricketts, M., Page, C. G., Ilovaisky, S. A., Chevalier, C., 1983, A\&A, 119, 171.

Narayan, R. \& Yi, I., 1995, ApJ, 452, 710.

Nowak, M. A., 2000, MNRAS, in press, astro-ph/0005232

Patterson. J.. 2000. IAUC $7413 ;$ see also http://www.astro.bio2.edu/cba.

Psaltis, D., Belloni, T. \& van der Klis, M., 1999. ApJ, 520, 262.

Psaltis, D. \& Norman, C., 2000, ApJ, in press, astro-ph/0001391

Poutanen. J. \& Coppi, P., 1998, Phys. Scripta, T77, 57 (astroph/9711316).

Quataert, E. \& Narayan, R., 1999, ApJ, 520, 298.

Remillard R., Morgan E., Smith D, Smith E. 2000, IAUC 7389

Revnivtsev M.. Sunvaev R. \& Borozdin K., 2000, submitted to A\&AL, astro-ph/0005212.

Stella, L., Vietri, M. \& Morsink, S., 1999, ApJ, 524, L63.

Uemura M., Kato T. \& Yamaoka H. 2000, IAUC 7390.

Wilson C. \& McCollough M. 2000, IAUC 7390

Yamaoka, K, Ueda, Y. \& Dotani, T., 2000, IAUC 7427.

Wardziński, G. \& Zdziarski, A. A., 2000, MNRAS, 314, 183.

Wilms, J., Nowak, M. A., Dove, J. B., Fender,R. P., \& Di Matteo, T., 1999, ApJ, 522, 460.

Zdziarski, A. A., Poutanen, J., Mikolajewska, J., Gierliński, M., Ebisawa, K., \& Johnson, W. N., 1998, MNRAS, 301, 435.

This paper has been produced using the Royal Astronomical Society/Blackwell Science $\mathrm{LT}_{\mathrm{E}} \mathrm{X}$ style file. 